PHYSICAL REVIEW E 95, 019905(E) (2017)

\title{
Publisher's Note: Asymptotic self-restabilization of a continuous elastic structure [Phys. Rev. E 94, 063005 (2016)]
}

F. Bosi, D. Misseroni, F. Dal Corso, S. Neukirch, and D. Bigoni

(Received 19 January 2017; published 30 January 2017)

DOI: 10.1103/PhysRevE.95.019905

This paper was published online on 27 December 2016 with typographical errors in an author footnote and in Eq. (9). D. Bigoni’s email address in the footnote should read as “davide.bigoni@unitn.it”. Equation (9) should read as

$$
F_{\mathrm{c}}=\frac{M^{2}\left(l_{\mathrm{in}}(t), t\right)}{2 B},
$$

The footnote and equation have been corrected as of 19 January 2017. The footnote and equation are incorrect in the printed version of the journal. 